\title{
I tempi sono maturi
}

\author{
The time is ripe
}

\author{
Cesare Greco
}

Monaldi Arch Chest Dis 2014; 82: 3-4.

UOC Cardiologia III - Azienda Complesso Ospedaliero S Giovanni Addolorata-Roma

Corresponding author: Dott. Cesare Greco; Viale Parioli 47; I-00197 Roma, Italy; E-mail address: cgreco@hsangiovanni.roma.it

"La riabilitazione cardiologica è un programma di prevenzione secondaria con l'esercizio fisico come base di un intervento che comprende programmi educazionali, controllo dei fattori di rischio cardiovascolare e ricerca dell'adozione spontanea di uno stile di vita sano da parte del paziente".

Questa semplice e sintetica definizione della Società Europea di Cardiologia ha guidato il cammino di questi due anni. Già da tempo la cardiologia riabilitativa (CR) italiana cercava di uscire da una visione minoritaria e quasi autoreferenziale in cui, sulla base di linee guida internazionali non adattate alla realtà nazionale, aveva reclamato l'invio di tutti i pazienti cardiopatici in fase post acuta nelle proprie strutture, senza peraltro ottenerlo in alcun modo. Già nel 2011 un primo Documento di consenso redatto da una commissione intersocietaria ANMCO-GICR aveva introdotto il criterio della priorità dell'accesso alle strutture degenziali di CR per i pazienti ad alto rischio, storicizzando e quindi superando il criterio della semplice indicazione a questo percorso assistenziale. Era però necessario compiere un altro importante passo avanti. Con la Conferenza di Consenso su "L'organizzazione dell'assistenza alla fase post acuta delle sindromi coronariche", tenutasi a Napoli nell'ottobre 2013 GICR-IACPR ha gettato le basi per la pubblicazione di un secondo Documento di Consenso intersocietario, questa volta sotto l'egida di ANMCO-GICR-GISE e con l'endorsement di ARCA, ANCE e SIMMG. Il Documento ha significativamente stabilito il record di reprint nella storia del Giornale Italiano di Cardiologia, elemento che sottolinea la grande attenzione del mondo cardiologico italiano verso un problema emergente: quello dell'assistenza alla fase post acuta delle malattie cardiache. Gli elementi più significativi di questo lungo lavoro sul post SCA sono due: 1) l'analisi delle mutazioni dell'epidemiologia clinica dell'infarto miocardico, compiuta grazie alla collaborazione con il Centro Nazionale di Epidemiologia, Sorveglianza e Promozione della Salute, Istituto Superiore di Sanità, Roma nell'ambito della convenzione stipulata con GICR-IACPR nel 2012,2) la condivisione unanime di una organizzazione dei flussi dell'assistenza nella fase post acuta che vede un impiego crescente di risorse con il crescere della complessità clinica.

Questi due elementi hanno di fatto posto la cardiologia riabilitativa al centro del dibattito sulla continuità delle cure cardiologiche ed all'interno del disegno di una rete assistenziale per la fase post acuta della SCA, rete da modellare a seconda del contesto locale.

Va però sottolineato come l'azione della CR e della sua società scientifica non debba limitarsi al solo scenario del day after sindromi coronariche acute. Vi sono due grandi campi in cui di fatto le nostre strutture sono quotidianamente impegnate: quello dello scompenso cardiaco e quello della fibrillazione atriale. Mentre alcune delle strutture più avanzate della CR nazionale sono da tempo con successo attive nel primo settore, è però mancata un'iniziativa generale di GICR-IACPR nel campo dello scompenso cardiaco, in cui è stata dimostrata la capacità dei programmi riabilitativi di ridurre significativamente la piaga delle reospedalizzazioni. Analogamente, mentre molti dei pazienti post cardiochirurgici soffrono di complicanze tachiaritmiche, la nostra società scientifica non ha affrontato i problemi e le sfide che la disponibilità di nuovi trattamenti anticoagulanti ha posto. Si tratta di un vuoto da riempire.

Sarebbe ingiusto limitare però solo ai più grandi temi di dibattito scientifico l'analisi dell'attività degli ultimi anni. Lo spirito di iniziativa dei componenti del Direttivo già per due volte, con Think Pad e Think PAD reloaded, ha indicato la metodologia e la strada da percorrere su tematiche apparentemente ai margini dell'attività di $\mathrm{CR}$, ma in realtà con un enorme ruolo potenziale come l'arteriopatia periferica.

Con il progetto Cardio Pain si è toccata per la prima volta una tematica così rilevante, quella del dolore, da essere regolata da un disposto legislativo specifico e tanto frequente nel periodo riabilitativo dopo un intervento cardochirurgico.

Isyde 2013 Directory, portato a termine insieme a SICOA ha fornito una mappa aggiornata delle strutture di CR e dato indicazioni preziose circa la loro struttura e la loro attività, ponendo le basi per una futura nuova survey. 
Negli ultimi due anni GICR-IACPR ha patrocinato più di quaranta congressi e ne ha organizzati più di venti a livello regionale, oltre all'appuntamento tradizionale di Cardioprevent 2013.

Nello stesso periodo si è tenuta la prima Convention dei fisioterapisti ed in occasione del Congresso di Genova si terrà la prima Convention degli infermieri della CR, a testimonianza del contributo di passione e cultura che le Aree non mediche portano alla nostra società.

È però indubbio che ci aspetta una nuova grande sfida: quella di fungere da raccordo tra le strutture cardiologiche per acuti ed il territorio. Si tratta di un ruolo che ci è connaturale sia dal punto di vista culturale che organizzativo e che finora nessuna società scientifica e nessuna iniziativa sono riuscite a riempire. Non sarebbe sorprendente se, anche in questo caso, GICR-IACPR riuscisse ad stimolare la comunità cardiologica e la medicina di base ad intraprendere il percorso virtuoso di appropriatezza della rete del post acuto e cronico, già a suo tempo progettata con lungimiranza dall'ANMCO nel documento Struttura ed organizzazione funzionale della cardiologia. I tempi sono maturi. 